\title{
Editorial
}

\section{Artificial Intelligence Applications in Biomedicine}

\author{
Panayiotis Vlamos, ${ }^{1}$ Konstantinos Lefkimmiatis, ${ }^{2}$ Catalina Cocianu, ${ }^{3}$ \\ Luminita State, ${ }^{4}$ and Zhiyuan Luo ${ }^{5}$ \\ ${ }^{1}$ Department of Informatics, Ionian University, Palaia Anaktora, 49100 Corfu, Greece \\ ${ }^{2}$ Brigham and Women's Hospital and the VA Boston Healthcare System, Harvard Medical School, West Roxbury VAMC, \\ 1400 VFW Parkway Room 2B111, West Roxbury, MA 02132, USA \\ ${ }^{3}$ Department of Informatics in Economy, Faculty of Economic Cybernetics, Statistics and Informatics, Bucharest Academy of \\ Economic Studies, 15-17 Calea Dorobantilor, District 1, 71131 Bucharest, Romania \\ ${ }^{4}$ Department of Mathematics and Computer Science, University of Pitesti, Street Targu din Vale, No. 1, Arges, 110040 Pitesti, Romania \\ ${ }^{5}$ Department of Computer Science, Royal Holloway University of London, Egham, Surrey TW20 0EX, UK
}

Correspondence should be addressed to Panayiotis Vlamos; vlamos@ionio.gr

Received 22 November 2012; Accepted 22 November 2012

Copyright ( 12013 Panayiotis Vlamos et al. This is an open access article distributed under the Creative Commons Attribution License, which permits unrestricted use, distribution, and reproduction in any medium, provided the original work is properly cited.

"Every aspect of learning or any other feature of intelligence can be so precisely described that a machine can be made to simulate it."-The Dartmouth Summer Research Project on Artificial Intelligence.

Recent technological advances in medical informatics and biomedicine facilitated the development of complex biomedical systems including innovated clinical and computer-based decision support systems, knowledge acquisition and management, medical imaging, computational intelligence in bioclinical medicine, molecular medicine, and healthcare organizational aspects. Artificial intelligence (AI) has a great impact on the fields of biology, biotechnology, and medicine in general and can be implemented in real world applications through machine learning techniques, neural computing, expert systems, fuzzy logic, genetic algorithms or Bayesian modelling. This issue of Artificial Intelligence Applications in Biomedicine (AIAB) compiles five innovating research articles, concerning machine learning methodologies, AI support systems in patient monitoring, genetic algorithms as well as optimization techniques and semantics.

The problem of classification, prediction, and diagnostic (CPD) errors is addressed in E. Eiland et al. paper, as well as the importance of relative class size (rCS) to end user problems. Authors made the first step in identifying the structure of CPD problems faced by end users and described the way that CPD evaluation measures and tools are relevant to end users. They have also identified measures that are efficacious for end users and shown how joint probability table (JPT) normalization and JPT tuning are useful for end user CPD evaluation.

A. Alexiou and P. Vlamos designed a new genetic algorithm (GA) for the representation of mitochondrial population through the framework of cultural algorithms (CA). It is well known that mitochondrial dysfunctions are highly associated with neurodegenerative diseases and related disorders; therefore, authors provide a combined simulation procedure for the optimization problem of ATP production in cells. The proposed algorithm is related to the main mitochondrial dynamics of complete fusion, transient fusion, fission and motility and the way that these operations can affect healthy mitochondrial population as an early symptom of neurodegenerative diseases.

An integrated system based on web applications, smartphones and an interconnection to Microsoft HealthVault platform is developed from E. Sourla et al. for monitoring chronic cardiology patients and early notifying and optimizing the process management of an emergency cardiologic incident. The decision system (DSS) supports cardiologic patient modules and is based on common cardiology diseases 
and fuzzy logic. The proposed AI system, CardioSmart365 is a complex expert system that combines DSS and web services, concerning patients, medical doctors, everyday clinical practice, research and science, and Healthcare Systems.

Additionally, the basin hopping $(\mathrm{BH})$ framework has been explored in A. Shehu et al. research paper, as a powerful global optimization tool for systems with multiple variables and modalities like biological macromolecules (proteins). Authors took into consideration different applications of importance in computational structural biology in order to demonstrate the importance of $\mathrm{BH}$ framework and specific characteristics such as adjacency of local minima and its relation to the quality of the reported global minimum.

Finally Z. Majkic extended both Montague's and Bealer's approaches for the intentional first-order logic (FOL) and design also a new extensional algebra for the FOL and the commutative homomorphic diagram that express the generalization of the Tarskian theory of truth for the FOL into the Frege/Russell's theory of meaning.

In conclusion, we hope these papers to enrich our readers and researchers in both theoretical and applied modern scientific fields, with respect to the strong relationship between artificial intelligence, information technology and mathematical modelling in biology and medicine.

Panayiotis Vlamos Konstantinos Lefkimmiatis Catalina Cocianu Luminita State Zhiyuan Luo 

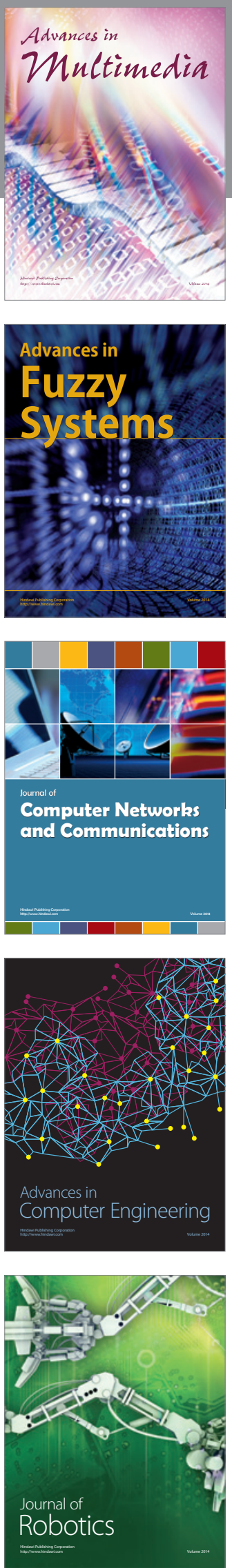

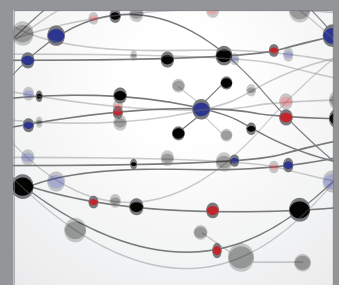

The Scientific World Journal
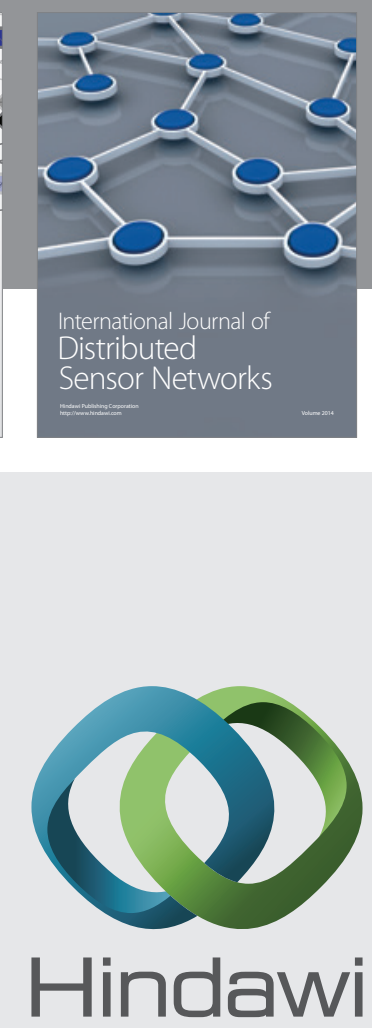

Submit your manuscripts at

http://www.hindawi.com
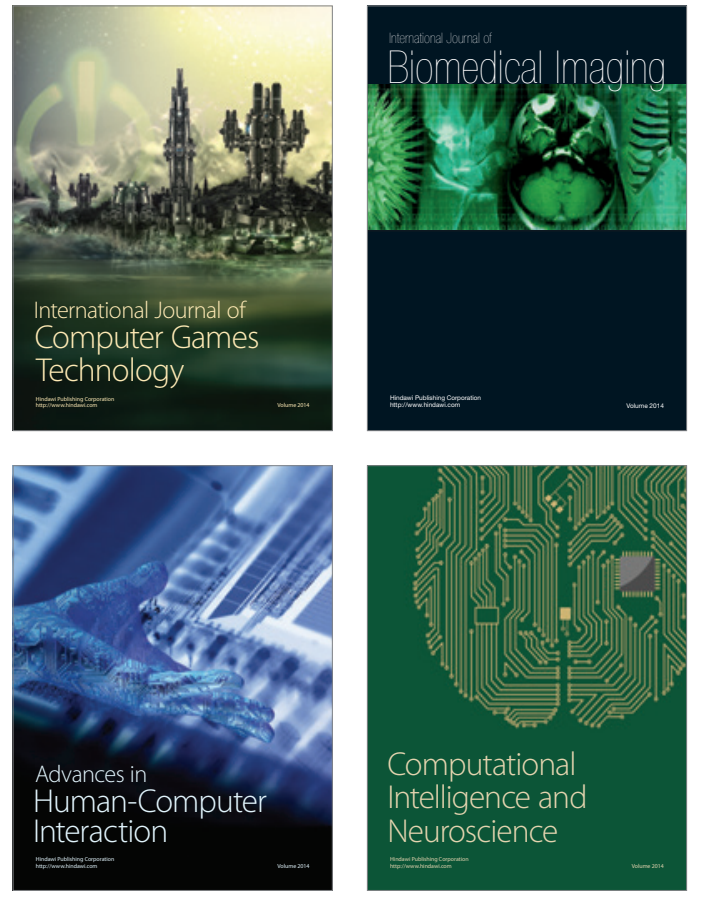
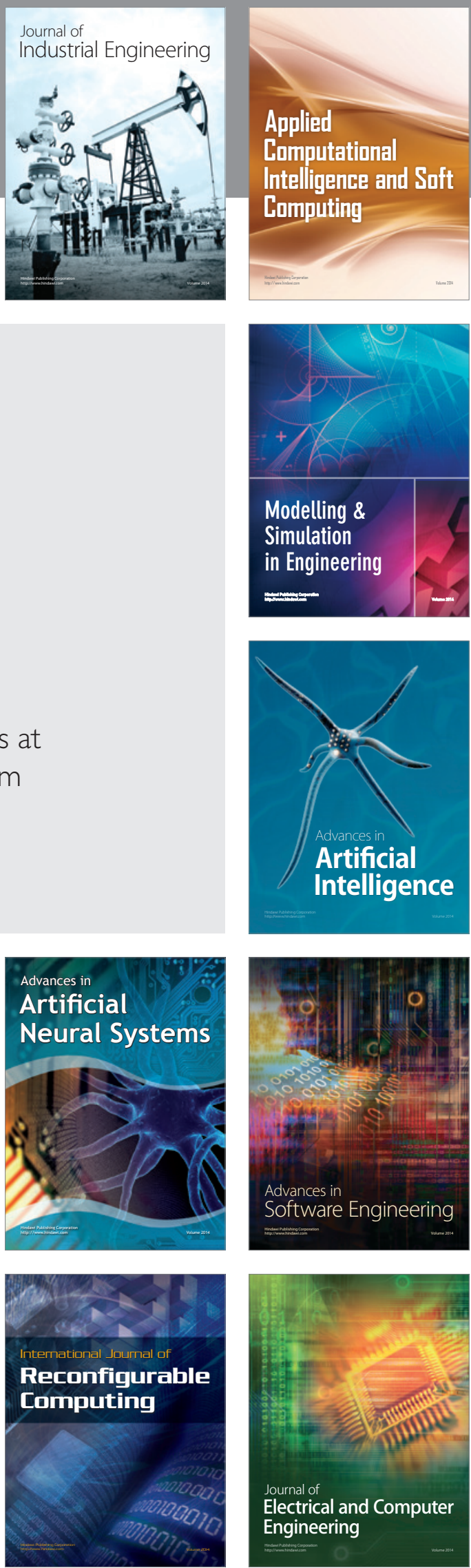